doi:10.1017/S1041610221002726

\section{The effect of Alzheimer's disease comorbidity in tap test response in idiopathic normal pressure hydrocephalus?}

We read with great interest the article by Kanemoto et al. (2021) in which they investigate the possible effect of Alzheimer's disease (AD) comorbidity in the cerebrospinal fluid (CSF) tap test response in idiopathic normal-pressure hydrocephalus (iNPH) subjects. In conclusion of their study, they found that iNPH subjects without response to the tap test displayed CSF features of concomitant AD pathology in comparison to those with a positive response to the tap test. Considering the rarity of the related studies and a large number of patients included, the results are surely crucial. However, we think that there may be some additional comments for a better understanding of the study results.

The authors investigate the presence of $\mathrm{AD}$ pathology by evaluating the CSF levels of amyloid $\beta$ (A $\beta) 1-40, A \beta 1-42, A \beta 42 / 40$ ratio, and total tau. However, they also discuss that the concentration of $A B$ and total tau in the CSF of iNPH patients may not be the best biomarker for AD pathology in these patients. They refer to a previous study showing that CSF proteins including $A \beta$ and total tau, also differed between iNPH patients and healthy subjects (Chen et al., 2017). Supporting this observation, the neuronal degeneration in iNPH has been linked with the accumulation of neurotoxins such as $\beta$-amyloid and tau-protein that are hypothesized to occur due to impaired blood-brain barrier and reduced CSF clearance in these subjects (Kudo et al., 2000). On the other hand, in the study by Kanemoto et al. (2021), there are no supporting data regarding the clinical and neuroimaging signs of $\mathrm{AD}$ in included patients groups to support the evidence of CSF parameters of $\mathrm{AD}$. In the current state, the results of this study may also be interpreted like that a distinct subgroup of iNPH subjects with decreased $\mathrm{CSF} \mathrm{A} \beta 42 / 40$ ratio and increased tau may not respond well to the CSF tap test and these patients may rather present prominently with cognitive symptoms. Therefore, we wonder if the authors might include evidence regarding clinical and neuroimaging findings to support $\mathrm{AD}$ pathology which is critical in such a study design.

The authors suggest that comorbidity with $\mathrm{AD}$ may be one of the factors that mediate a negative response to the tap test in patients with iNPH. However, only the improvement of cognition was found to be related to a CSF A $\beta 42 / 40$ ratio. Besides, improvement in some of the cognitive measures, including scores of $\mathrm{FAB}$, delayed story recall of RBMT, and $\mathrm{A} / \mathrm{C}$ in WMS-R, which were not used to judge the response to the tap test, also occurred in the iNPH-nTT group following the tap test. They also mention that four subjects in the iNPH-nTT group received shunt surgery and three showed a significant improvement. Ergo, we think that the rationale behind the categorization of patients under the iNPH-pTT and iNPH-nTT groups should be evaluated meticulously while interpreting these results. The authors evaluate the response to the tap test according to the recommendation of Japanese guidelines (Mori et al., 2012). However, the cognitive subdomains are not evaluated separately in this guideline. In light of the above discussion, it is sensible to argue that the presence of $\mathrm{AD}$ pathology in iNPH might rather prevent the clear evaluation of CSF tap test response and lead to a false- negative interpretation. Therefore, it can be speculated that the response to CSF tap test in these patients with suspicion of $\mathrm{AD}$ pathology should be evaluated in a more detailed manner including the additional cognitive tests evaluating frontal lobe functions.

Of note, the basal cognitive scores in the iNPHnTT group were generally lower than those in the INPH-PTT group, whereas the scores regarding the gait and urinary function were rather better than scores of subjects in the INPH-PTT group (Kanemoto et al., 2021, Table 1). Ergo, these results may also suggest that the patient presenting with an iNPH-like clinic with prominent cognitive symptoms may consider an underlying $\mathrm{AD}$ pathology. Therefore, we wonder if the authors may include also the results of the statistical analyses comparing these subdomains of the iNPH triads separately at presentation between the groups (iNPH-nTT and iNPH-pTT groups).

The clarification of these issues raised in this letter and the results of the future related studies may provide crucial contributions to our understanding of the clinical evaluation processes of iNPH and underlying pathophysiology. About $19 \%$ - and as much as $24 \%$ - of iNPH patients undergoing CSF shunt insertion have a neuropathologically confirmed diagnosis of $\mathrm{AD}$ (Golomb et al., 2000; Oliveira et al., 2019; Pomeraniec et al., 2016). 
Considering that there are a large number of neurodegenerative comorbidities reported in patients with iNPH-like clinics, multidirectional evaluation is required in these patients. Some authors draw attention to the neurodegenerative pathologies as a common accompanying entity in iNPH subjects, and they emphasize that long-term benefit may not be achieved after shunt surgery in this patient group (Hamilton et al., 2010). On the other hand, there is a tendency to support shunt surgery in iNPH patients with accompanying neurodegenerative disease, based on the clinical benefit provided by surgery (Golomb et al., 2000; Onder, 2021). Such that, Golomb et al. found that the biopsy-positive diagnosis of $\mathrm{AD}$ did not initially affect the beneficial effect of the shunt on the iNPH symptoms (Golomb et al., 2000). However, there is no randomized controlled study to evaluate the long-term effect of shunt surgery in iNPH patients with comorbid neurodegenerative disease. Although the results of this study (Kanemoto et al., 2021) may suggest unresponsiveness to CSF removal in subjects with AD comorbidity, the detailed analyses of the study (in light of the comments in our letter) may rather suggest the opposite view. Future studies including more precise documentation of the $\mathrm{AD}$ pathology and data regarding the post-shunt clinical outputs are warranted to clarify these discussions. Finally, the reliability of the current guidelines (Mori et al., 2012) in evaluating the decision to CST tap test, a gold standard method in deciding shunt surgery, may be reevaluated in future comprehensive studies including subjects with distinct clinical scenarios such as accompanying $\mathrm{AD}$ pathology.

\section{Conflict of interest}

None.

\section{Description of authors' roles}

Concept - H.O.; design - H.O.; supervision - H.O., S.C.; materials - H.O; data collection and/or processing - H.O, B.K.; analysis and/or interpretationHO., S.C.; literature search - H.O, B.K.; writing manuscript - H.O., B.K., critical review H.O., S.C.

\section{References}

Chen, Z., Liu, C., Zhang, J., Relkin, N., Xing, Y. and Li, Y. (2017). Cerebrospinal fluid Abeta42, t-tau, and p-tau levels in the differential diagnosis of idiopathic normalpressure hydrocephalus: a systematic review and metaanalysis. Fluids and Barriers of the CNS, 14, 13.

Golomb, J. et al. (2000). Alzheimer's disease comorbidity in normal pressure hydrocephalus: prevalence and shunt response. Fournal of Neurology, Neurosurgery \& Psychiatry, $68,778-781$.

Hamilton, R. et al. (2010). Lack of shunt response in suspected idiopathic normal pressure hydrocephalus with Alzheimer disease pathology. Annals of Neurology, 68, 535-540.

Kanemoto, H. et al. (2021). Cerebrospinal fluid amyloid beta and response of cognition to a tap test in idiopathic normal pressure hydrocephalus: a case-control study. International Psychogeriatrics, 1-9. DOI 10.1017/ S1041610221000661 (Online ahead of print).

Kudo, T. et al. (2000). Tau protein is a potential biological marker for normal pressure hydrocephalus. Psychiatry and Clinical Neurosciences, 54, 199-202.

Mori, E. et al. (2012). Guidelines for management of idiopathic normal pressure hydrocephalus: second edition. Neurologia medico-chirurgica, 52, 775-809.

Oliveira, L. M., Nitrini, R. and Roman, G. C. (2019). Normal-pressure hydrocephalus: a critical review. Dementia E Neuropsychologia, 13, 133-143.

Onder, H. (2021). The sustained improvement after lumbar puncture in an idiopathic normal pressure hydrocephalus subject with synucleinopathy. Neurological Sciences. DOI 10.1007/s10072-021-05535-1 (ahead of print).

Pomeraniec, I. J., Bond, A. E., Lopes, M. B. and Jane, J. A., Sr (2016). Concurrent Alzheimer's pathology in patients with clinical normal pressure hydrocephalus: correlation of high-volume lumbar puncture results, cortical brain biopsies, and outcomes. Fournal of Neurosurgery, 124, 382-388.

Halil Onder, (i) Bilge Kocer and Selcuk Comoglu

Neurology Clinic, Diskapi Yildirim Beyazit Training and Research Hospital, Ankara, Turkey

Correspondence should be addressed to: Halil Onder, Neurology Clinic, Diskapi Yildirim Beyazit Training and Research Hospital, Şehit Ömer Halisdemir Street. No: 20 Altındag, Ankara 06110, Turkey. Phone: 03125962000. Fax: 0 (312) 596-2000. Email: halilnder@yahoo.com 